\title{
Intratumor heterogeneity of prognostic DNA-based molecular markers in adrenocortical carcinoma
}

\author{
Anne Jouinot ${ }^{1,2}$, Juliane Lippert ${ }^{3}$, Martin Fassnacht ${ }^{3,4}$, Bruno de La Villeon ${ }^{1}$, Amandine Septier ${ }^{1}$, Mario Neou ${ }^{1}$, \\ Karine Perlemoine ${ }^{1}$, Silke Appenzeller ${ }^{3}$, Mathilde Sibony ${ }^{1,5}$, Sébastien Gaujoux ${ }^{1,6}$, Bertrand Dousset ${ }^{1,6}$, \\ Rossella Libe ${ }^{1,2}$, Lionel Groussin ${ }^{1,2}$, Cristina L Ronchi ${ }^{3,7,8}$, Guillaume Assié ${ }^{1,2}$ and Jérôme Bertherat ${ }^{1,2}$ \\ 'Université de Paris, Institut Cochin, INSERM U1016, CNRS UMR8104, Paris, France \\ ${ }^{2}$ Department of Endocrinology, Assistance Publique Hôpitaux de Paris, Hôpital Cochin, Paris, France \\ ${ }^{3}$ Department of Internal Medicine I, Division of Endocrinology and Diabetes, University Hospital, University of Wuerzburg, Wuerzburg, Germany \\ ${ }^{4}$ Comprehensive Cancer Center Mainfranken, University of Wuerzburg, Wuerzburg, Germany \\ ${ }^{5}$ Department of Pathology, Assistance Publique Hôpitaux de Paris, Hôpital Cochin, Paris, France \\ ${ }^{6}$ Department of Digestive and Endocrine Surgery, Assistance Publique Hôpitaux de Paris, Hôpital Cochin, Paris, France \\ ${ }^{7}$ Institute of Metabolism and System Research, University of Birmingham, Birmingham, UK \\ ${ }^{8}$ Centre for Endocrinology, Diabetes and Metabolism, Birmingham Health Partners, Birmingham, UK
}

Correspondence should be addressed to J Bertherat: jerome.bertherat@aphp.fr

\section{Abstract}

Background: The prognosis of adrenocortical carcinoma (ACC) is heterogeneous. Genomic studies have identified ACC subgroups characterized by specific molecular alterations, including features measured at DNA level (somatic mutations, chromosome alterations, DNA methylation), which are closely associated with outcome. The aim of this study was to evaluate intratumor heterogeneity of prognostic molecular markers at the DNA level. Methods: Two different tissue samples (primary tumor, local recurrence or metastasis) were analyzed in 26 patients who underwent surgery for primary or recurrent ACC. DNA-related biomarkers with prognostic role were investigated in frozen and paraffinembedded samples. Somatic mutations of $\mathrm{p} 53 / \mathrm{Rb}$ and $\mathrm{Wnt} / \mathrm{\beta}$-catenin pathways were assessed using next-generation sequencing $(n=26)$, chromosome alteration profiles were determined using SNP arrays $(n=14)$ and methylation profiles were determined using four-gene bisulfite pyrosequencing $(n=12)$.

Results: Somatic mutations for ZNRF3, TP53, CTNN1B and CDKN2A were found in 7, 6, 6 and 4 patients, respectively, with intratumor heterogeneity in $8 / 26$ patients (31\%). Chromosome alteration profiles were 'Noisy' (numerous and anarchic alterations) in $8 / 14$ and 'Chromosomal' (extended patterns of loss of heterozygosity) in 5/14 of the study samples. For these profiles, no intratumor heterogeneity was observed. Methylation profiles were hypermethylated in 5/12 and non-hypermethylated in 7/12 of the study samples. Intratumor heterogeneity of methylation profiles was observed in $2 / 12$ patients (17\%).

Conclusions: Intratumor heterogeneity impacts DNA-related molecular markers. While somatic mutation can differ, prognostic DNA methylation and chromosome alteration profile seem rather stable and might be more robust for the prognostic assessment. 


\section{Introduction}

Adrenocortical carcinoma is a rare cancer with poor but heterogeneous prognosis. The 5-year overall survival of ACC patients remains below $40 \%$ in most series (1).

The main prognostic factors used in clinical practice at present are the tumor extension, best reflected by the ENSAT stage (2), and the tumor proliferation, estimated either by mitotic count (3) or Ki67 proliferation index (4, 5). However, the prognosis still varies widely among tumors with the same tumor stage and proliferation index (6).

Recently, pan-genomic studies have identified molecular subtypes closely associated with prognosis (7, 8). A first subtype associates a ' $C 1 \mathrm{~A}^{\prime}$ transcriptome profile, characterized by upregulation of proliferative genes, a CpG island hypermethylation, a 'Noisy' chromosome alteration profile - that is, numerous and anarchic alterations - and an accumulation of mutations in $\mathrm{p} 53 / \mathrm{Rb}$ and $\mathrm{Wnt} / \beta$-catenin-related genes. This subgroup is associated with very poor outcome. Conversely, another subtype of ACC associates a ' $\mathrm{C} 1 \mathrm{~B}$ ' transcriptome profile, enriched in immune-related genes, no hypermethylation, a 'Chromosomal' genome profile - that is, extended patterns of loss of heterozygosity ( $\mathrm{LOH}$ - and a low mutation rate. This subgroup is associated with a better outcome. Hence, targeted molecular markers have been proposed to complete the prognostic assessment of ACC $(9,10,11)$. These markers are either measured at the tumor DNA level, including somatic mutations, chromosome alteration and DNA methylation profile, or at the RNA level, including transcriptome and targeted gene expression profiles.

Intratumor heterogeneity of somatic mutations has been reported in many cancer types $(12,13,14)$. In a small series of exome sequencing in 14 ACC patients, intratumor heterogeneity was reported in $43-63 \%$ of somatic mutations among different metastatic sites from the same patient (15). In addition to somatic mutations, intratumor heterogeneity of DNA methylation has been reported in several cancer types $(16,17)$. Whether molecular alterations identified as major prognostic features of ACC are exposed to intratumor heterogeneity remains to be determined.

The aim of our study was to assess the robustness of targeted molecular markers measurable at the DNA level - somatic mutations, chromosome alteration profile and targeted DNA methylation profile - by performing multiple measures for different tumor manifestations in the same patients. Variability of the prognostic molecular assay was determined for each measurement.

\section{Materials and methods}

\section{Tumor samples}

Two cohorts of ACC patients were included in this study: 14 patients from Cochin Hospital, Paris, France, and 12 patients from Wuerzburg University Hospital, Germany. Tissue samples were obtained from two different tumor sites for each patient (Supplementary Table 1, Supplementary Fig. 1, see section on supplementary materials given at the end of this article), including primary tumor (P), local recurrence (R) or distant metastasis $(\mathrm{M})$. Tumor specimens were collected between 2001 and 2015 and were either frozen in the Cochin cohort or formalin-fixed paraffinembedded (FFPE) samples in the Wuerzburg cohort, as previously described $(9,11)$. The diagnosis of ACC was confirmed by an expert endocrine pathologist, according to Weiss criteria (18).

Written informed consent for the molecular analysis and the collection and use of the clinical data was obtained from all patients and the study was approved by the Comite de protection des personnes Ile de France 1 (application \#13311) and the Ethics Committee of the University of Würzburg (registration number 88/11).

\section{DNA isolation}

Tumor DNA was extracted and purified by proteinase $\mathrm{K}$ digestion and ethanol extraction, followed by a clean-up step on columns (Qiagen) in Cochin cohort and with the GeneRead DNA FFPE Kit (Qiagen) in Wuerzburg cohort, according to the manufacturer's protocols. The quality of DNA was analyzed by the Nanodrop ND-1000 spectrophotometer (Nyxor Biotech) in Cochin cohort and by the GeneRead DNA QuantiMIZE Assay Kit (Qiagen) in Wuerzburg cohort.

Leukocyte DNA was isolated with the DNA isolation kit for mammalian blood (Roche) in Cochin cohort and with the NucleoSpin Blood L Kit (Macherey-Nagel, Bethlehem, PA, USA) in Wuerzburg cohort, according to the manufacturer's instructions.

\section{Sequence mutations and copy number alterations}

Somatic alterations in $\mathrm{p} 53 / \mathrm{Rb}(T P 53, R B 1, C D K 4$, and $C D K N 2 A)$ and Wnt/ $\beta$-catenin (CTNNB1, and ZNRF3) pathways were determined in both cohorts $(n=26)$.

For each patient, two samples from two different tumor regions were studied.

In the Cochin cohort, sequence variants were called using exome sequencing (HiSeq, Illumina) for 4/28 samples

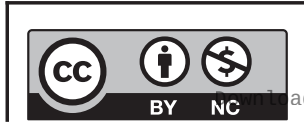

This work is licensed under a Creative Commons Attribution-NonCommercial 4.0 International License. ded from Bioscientifica.com at 04/26/2023 11:33:54AM 
and targeted next-generation sequencing (NGS) with a custom panel (Ampliseq and PGM, Life Technologies) for 24/28 samples, as previously reported (11).

In the Wuerzburg cohort, sequence variants were called using targeted NGS with the GeneRead DNAseq Human Comprehensive Cancer Panel V2 and the GeneRead DNAseq Panel PCR Kit V2 (Qiagen and NextSeq500, Illumina) and Sanger sequencing for ZNRF3 for 20/24 samples, as previously reported (9), and with a QIAseq Targeted DNA Custom Panel (Qiagen and NextSeq500, Illumina) for $4 / 24$ samples.

Sequence mutations were defined as somatic variants that fulfill the following criteria: coverage $>100 \times$ in targeted NGS or $>30 \times$ in exome sequencing, rare in population database ( $<0.02$ in Exome Aggregation Consortium), allelic ratio $>0.2$, exonic or splicing, nonsynonymous, and not predicted as benign by at least one prediction algorithm including SIFT (19), Popyphen2 (20) and MutationTaster2 (21). All nonsynonymous somatic variants were validated visually using the Integrative Genomics Viewer (22) or GensearchNGS (PhenoSystems®, Braine le Chateau, Belgium). In case of subclonal variant - called in one tumor region but not in the other - the coverage depth of the negative region was also checked. Comparison of single nucleotide polymorphisms genotypes was used to verify the proper matching of the two samples for each patient (data not shown).

In addition to sequence variations, copy number alterations were called by analyzing SNP array data in the Cochin cohort $(n=14)$. Homozygous deletions and amplifications were called if log R ratio (LRR) was lower or higher than three-fold the s.D., respectively (11).

Calculation of recurrence rate was performed in both cohorts $(n=26)$ for genes usually subject to sequence mutations (TP53, CTNNB1, RB1) and in the Cochin cohort only $(n=14)$ for genes mostly subject to copy number alterations (ZNRF3, CDKN2A, CDK4).

\section{Chromosome alterations}

Chromosome alteration profiles were determined in the Cochin cohort only $(n=14)$ using Infinium HumanOmniExpress and HumanCore SNP arrays (Illumina).

Abnormal chromosome segments were generated with Genome Alteration Print (23), then filtered and smoothed. Filters included a minimal size, at least 20 SNPs with germline heterozygosity; a reduced noise within the segment, measured by the s.D. of mirrored B-allele frequency (MBAF) (a value between 0.5 and 1, reflecting the allelic ratio), expected lower than the s.D. of MBAF from normal chromosomes; and a validated segment the MBAF generated by GAP was expected close (no more or less than 0.2) to the computed MBAF of the segment. Smoothing was performed by merging any consecutive segments with close allelic ratios - defined by MBAF values lower than the s.D. of MBAF from normal chromosomes.

Each sample was assigned to 'Noisy' profile, that is, numerous and anarchic chromosome alterations; 'Chromosomal', that is, extended LOH; or 'Quiet', a limited number of chromosome alterations, following a classification rule based on the alterations of nine chromosome arms (1p, 1q, 2p, 2q, 11p, 11q, 18p, 18q, $22 q)$, as previously reported $(8,11)$. A sample was called 'Chromosomal' if at least seven chromosome arms presented complete LOH; a sample was called 'Quiet' if less than four chromosome arms were altered; and a sample was called 'Noisy' if 20 or more altered chromosome segments were identified (11).

\section{Targeted DNA methylation analysis}

Methylation profiles were determined in the Wuerzburg cohort only ( $n=12)$, using bisulfite pyrosequencing of four genes (PAX5, PAX6, PYCARD, and GSTP1), as previously reported $(9,24)$. The samples were classified as 'Not hypermethylated' if the mean methylation of all assays of all genes was $\leq 25 \%$. If the mean methylation was $>25 \%$, the samples were categorized as 'Hypermethylated'.

\section{Statistical analysis}

Calculations were performed using $\mathrm{R}$ statistical software (25).

Comparisons between groups were assessed using Mann-Whitney Wilcoxon test for quantitative variables and Fisher's test for qualitative variables. Paired tests were used for the comparison of the matched tumor samples.

Overall survival was defined as the time elapsed between surgery of the primary tumor and death or last follow-up visit.

All $P$-values were two-sided, and the level of significance was set at $P<0.05$.

\section{Results}

\section{Patient characteristics}

Patient characteristics are presented in Table 1. 
Table 1 Patients characteristics.

\begin{tabular}{|c|c|c|c|c|}
\hline Characteristics & Total & Cochin cohort & Wuerzburg cohort & P-value \\
\hline Sex & & & & 0.02 \\
\hline Female & 11 & 9 & 2 & \\
\hline Male & 15 & 5 & 10 & \\
\hline \multirow[t]{2}{*}{ Age (years) } & & & & 0.49 \\
\hline & $44(18-76)$ & $49(24-76)$ & $44(18-67)$ & \\
\hline ENSAT stage at diagnosis & & & & 0.15 \\
\hline 1 & 4 & 4 & 0 & \\
\hline II & 10 & 4 & 6 & \\
\hline III & 6 & 2 & 4 & \\
\hline IV & 6 & 4 & 2 & \\
\hline \multirow[t]{2}{*}{ Weiss score } & & & & 0.73 \\
\hline & $6(2-9)$ & $6(4-9)$ & $6(2-9)$ & \\
\hline \multirow[t]{2}{*}{ Ki67 index (\%) } & & & & 0.27 \\
\hline & $10(0-70)$ & $9(0-70)$ & $12(2-40)$ & \\
\hline Origin of samples & & & & 0.34 \\
\hline Primary/recurrence or metastasis & 20 & 9 & 11 & \\
\hline Primary/primary & 2 & 2 & 0 & \\
\hline Recurrence or metastasis/recurrence & 4 & 3 & 1 & \\
\hline Delay between surgeries of the two samples & & & & 0.005 \\
\hline Synchronous & 10 & 9 & 1 & \\
\hline Metachronous & 16 & 5 & 11 & \\
\hline
\end{tabular}

Results are expressed in median (range) for quantitative variables or numbers for qualitative variables.

Two different tumor samples were analyzed for each patient, including 24 primary tumor, 7 local recurrence and 21 distant metastasis.

Compared to the Cochin cohort, patients from the Wuerzburg cohort included more males (10/12 vs 5/14, $P=0.02)$ and more metachronous surgeries (11/12 vs $5 / 14, P=0.005)$. Age, ENSAT stage, Weiss score and Ki67 proliferation index were similar in the two cohorts.

Median OS was 40.2 months in Cochin cohort and 30.2 months in Wuerzburg cohort. Two patients died from post-operative complications. All other patients received adjuvant mitotane after the first surgery. No patient received chemotherapy or radiation therapy before surgery of primary tumor or metastases.

\section{Sequence mutations and copy number alterations profiles}

Sequence mutations were determined using NGS and Sanger sequencing in 26 patients, and copy number alterations were determined using SNP array in 14 patients.

Combining sequence mutations and copy number alterations, the most recurrently altered genes were $Z N R F 3$ (7/28 samples), CDKN2A (6/28 samples), TP53 (10/52 samples), and CTNNB1 (10/52 samples, Fig. 1, Table 2). No alteration of $C D K 4$ and $R B 1$ was observed. For $8 / 26$ patients, no alteration was identified in the two tumor samples.

Subclonal alterations were observed in $8 / 26$ patients (31\%) - 4/14 in the Cochin cohort and 4/12 in the
Wurzburg cohort - with one or several gene alterations found in one ACC region but not in the other (Fig. 1, Table 2, Supplementary Fig. 2), including ZNRF3 $(n=5)$, CDKN2A $(n=2), \operatorname{TP53}(n=3)$, and CTNNB1 $(n=2)$. For subclonal mutations - detected in only one ACC region the coverage depth of the negative region was at least $30 \times$.

\section{Chromosome alteration profiles}

Chromosome alterations were determined using SNP array in 14 patients (Fig. 2).

The percentage of tumor cells was estimated from SNP profiles and ranged from 40 to 100\% (median 80\%, Supplementary Table 2). The proportion of genome alteration ranged from 11.6 to $88.2 \%$. The proportion of altered genome was not significantly different between primary tumors (median 38.8\%) and matched local or metastatic recurrences (median 53.2\%, $P=0.30$, Supplementary Table 2). Intratumor heterogeneity of chromosome alterations was observed to a variable extent in all matched tumor samples (Fig. 2), and subclonal events could be identified within individual profiles (Supplementary Fig. 3).

However, beyond these subclonal focal events, the global chromosome alteration profile remained stable. More precisely, chromosome alteration profiles were classified as 'Noisy' for 16/28 samples and 'Chromosomal' for 10/28 samples. Neither the 'Noisy' nor the 'Chromosomal' status changed between the different

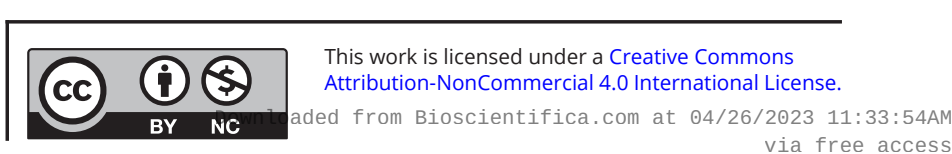




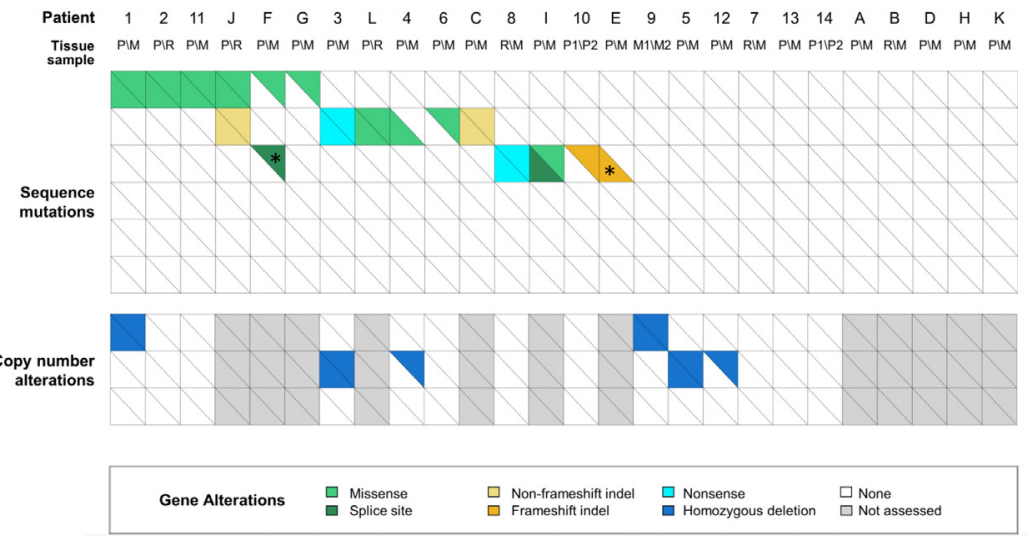

\section{Figure 1} CTNNB1 ZNFR3 CDKN2A $R B 1$ CDK4 ZNFR3 CDKN2A CDK4

Detection of somatic mutations in two distinct ACC regions (Cochin and Wuerzburg cohorts, 26 patients). Patients from the Cochin cohort are numbered from 1 to 14 and patients from the Wuerzburg cohort are designated by letters A-L. Sequence mutations and gene copy number alterations are depicted with color in matched ACC samples for $\mathrm{p} 53 / \mathrm{Rb}$ and $\mathrm{Wnt} / \mathrm{\beta}$-catenin genes. In each box, the lower left part represents the alterations of the first tumor sample and the upper right part represents the alterations of the second tumor sample. P, sample from primary tumor; $\mathrm{R}$, sample from local recurrence; $\mathrm{M}$, sample from metastasis. *Mutations identified by Sanger sequencing. tumor locations. Especially, no 'Noisy' subclone could be found in a 'Chromosomal' tumor. Of note, for the two samples of one patient, the chromosome alteration profiles showed no extended $\mathrm{LOH}$ and an intermediate number of chromosome segments and could not be reliably classified as 'Chromosomal', 'Noisy' or 'Quiet' profiles (Fig. 2, Supplementary Table 2).

\section{Methylation profiles}

Methylation profiles were determined using bisulfite pyrosequencing of four genes (PAX5, PAX6, PYCARD, and GSTP1) in 12 patients.

Average methylation of four genes ranged from 3 to $72 \%$ and was not significantly different between primary

Table 2 Somatic mutations in matched ACC samples (Cochin and Wuerzburg cohorts, 26 patients).

\begin{tabular}{|c|c|c|}
\hline Patient & Sample & Gene \\
\hline \multirow[t]{2}{*}{1} & $P$ & TP53 \\
\hline & $M$ & TP53 \\
\hline \multirow[t]{2}{*}{2} & $\mathrm{P}$ & TP53 \\
\hline & $\mathrm{R}$ & TP53 \\
\hline \multirow[t]{2}{*}{3} & $\mathrm{P}$ & CTNNB1 \\
\hline & $M$ & CTNNB1 \\
\hline 4 & $P$ & CTNNB1 \\
\hline 6 & $M$ & CTNNB1 \\
\hline \multirow[t]{2}{*}{8} & $\mathrm{R}$ & ZNRF3 \\
\hline & $\mathrm{M}$ & ZNRF3 \\
\hline 10 & $\mathrm{P} 2$ & ZNRF3 \\
\hline \multirow[t]{2}{*}{11} & $\mathrm{P}$ & TP53 \\
\hline & $M$ & TP53 \\
\hline \multirow[t]{2}{*}{ C } & $\mathrm{P}$ & CTNNB1 \\
\hline & $M$ & CTNNB1 \\
\hline E & $P$ & ZNRF3 \\
\hline \multirow[t]{3}{*}{$\mathrm{F}$} & $M$ & TP53 \\
\hline & $\mathrm{M}$ & TP53 \\
\hline & $M$ & ZNRF3 \\
\hline G & $M$ & TP53 \\
\hline \multirow[t]{2}{*}{ I } & $\mathrm{P}$ & ZNRF3 \\
\hline & $M$ & ZNRF3 \\
\hline \multirow[t]{4}{*}{ J } & $P$ & CTNNB1 \\
\hline & $P$ & TP53 \\
\hline & $\mathrm{R}$ & CTNNB1 \\
\hline & $\mathrm{R}$ & TP53 \\
\hline \multirow[t]{2}{*}{ L } & $P$ & CTNNB1 \\
\hline & $\mathrm{R}$ & CTNNB1 \\
\hline
\end{tabular}

\begin{tabular}{l} 
Alteration type \\
\hline Nonsynonymous SNV \\
Nonsynonymous SNV \\
Nonsynonymous SNV \\
Nonsynonymous SNV \\
Stopgain \\
Stopgain \\
Nonsynonymous SNV \\
Nonsynonymous SNV \\
Stopgain \\
Stopgain \\
Frameshift deletion \\
Nonsynonymous SNV \\
Nonsynonymous SNV \\
Non-frameshift deletion \\
Non-frameshift deletion \\
Frameshift deletion \\
Nonsynonymous SNV \\
Nonsynonymous SNV \\
Splice site SNV \\
Nonsynonymous SNV \\
Splice site SNV \\
Nonsynonymous SNV \\
Non-frameshift deletion \\
Nonsynonymous SNV \\
Non-frameshift deletion \\
Nonsynonymous SNV \\
Nonsynonymous SNV \\
Nonsynonymous SNV \\
\hline
\end{tabular}

\begin{tabular}{|c|c|}
\hline AA change & Allelic ratio \\
\hline p.C176F & 0.42 \\
\hline p.C176F & 0.64 \\
\hline p.R342P & 0.55 \\
\hline p.R342P & 0.53 \\
\hline p.Y30X & 0.39 \\
\hline p.Y30X & 0.48 \\
\hline p.S45F & 0.27 \\
\hline p.S45P & 0.44 \\
\hline p.Q167X & 0.54 \\
\hline p.Q167X & 0.86 \\
\hline p.A224fs & 0.77 \\
\hline p.R337C & 0.81 \\
\hline p.R337C & 0.93 \\
\hline p.S45del & 0.26 \\
\hline p.S45del & 0.64 \\
\hline p.E674Pfs*95 & 0.50 \\
\hline p.R248W & 0.36 \\
\hline p.R175H & 0.38 \\
\hline p.? & 0.66 \\
\hline p.R337c & 0.89 \\
\hline p.? & 0.50 \\
\hline p.P179L & 0.50 \\
\hline p.E267del & 0.30 \\
\hline p.Q245S & 0.81 \\
\hline p.E267del & 0.20 \\
\hline p.Q245S & 0.34 \\
\hline p.S45P & 0.44 \\
\hline p.S45P & 0.85 \\
\hline
\end{tabular}

$A A$, amino acid; $M$, sample from metastasis; $P$, sample from primary tumor; $R$, sample from local recurrence; SNV, single nucleotide variation; ?, uncertain impact in protein sequence.

Patients from the Cochin cohort are numbered from 1 to 14 and patients from the Wuerzburg cohort are designated by letters A-L.

https://ec.bioscientifica.com

https://doi.org/10.1530/EC-20-0228 (c) 2020 The authors Published by Bioscientifica Ltd

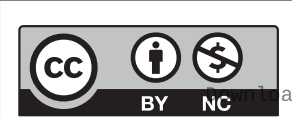

This work is licensed under a Creative Commons Attribution-NonCommercial 4.0 International License. ded from Bioscientifica.com at 04/26/2023 11:33:54AM 

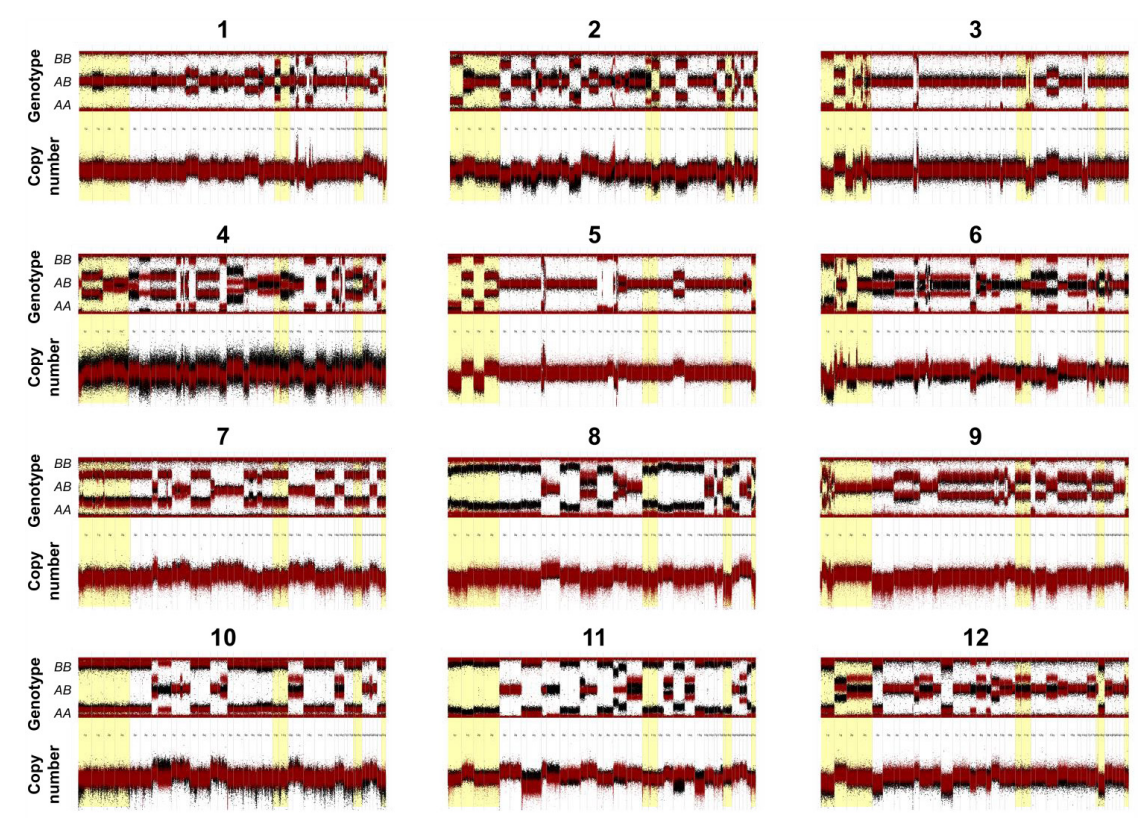

13
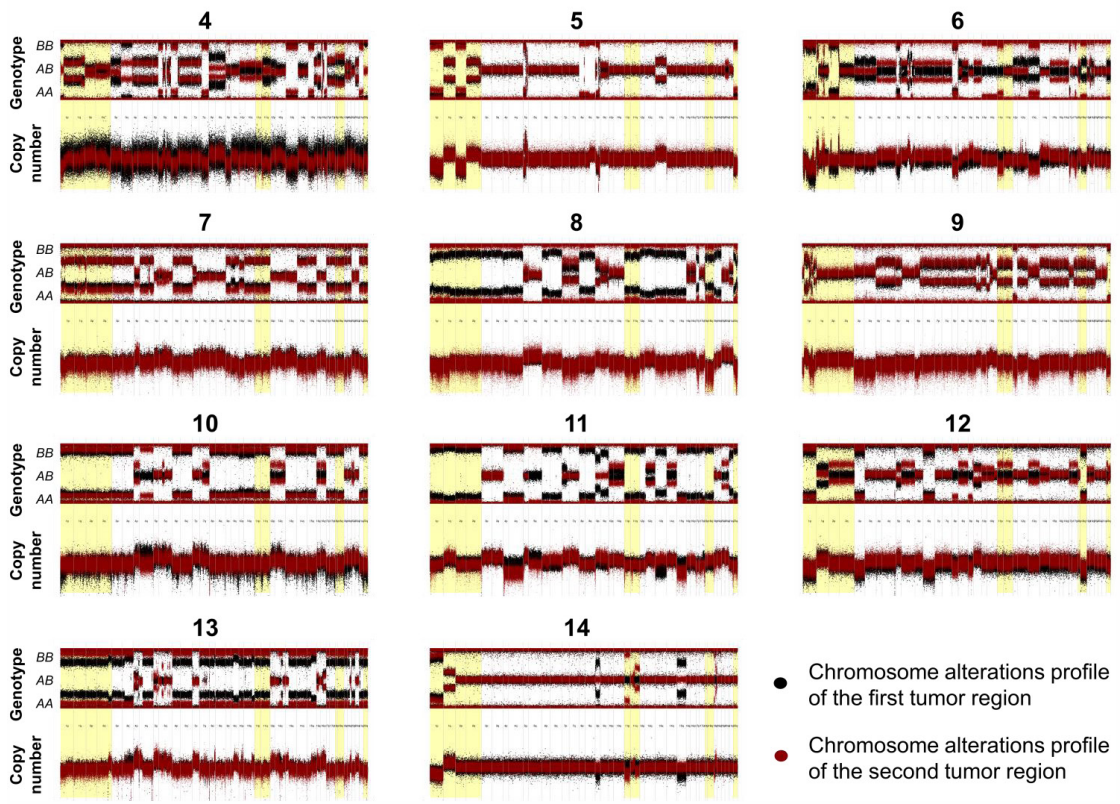

Chromosome alterations profile of the first tumor region

Chromosome alterations profile of the second tumor region

\section{Figure 2}

Superimposed chromosome alterations profiles in two distinct ACC regions (Cochin cohort, 14 patients). Superimposed chromosome alteration profiles are represented in black and red plots for each patient. Different samples within the same patient are predominantly homogenous, especially regarding the nine chromosome arms (in yellow) that were previously identified for classifying 'Chromosomal' or 'Noisy' prognostic profiles (11). tumor (median 31\%) and matched local or metastatic recurrence (median 36\%, $P=0.72$ ).

Methylation profiles were classified as 'Hypermethylated' in 10/24 samples and 'Not hypermethylated' for 14/24 samples (Fig. 3). Intratumor heterogeneity of methylation profile was observed in $2 / 12$ patients $(17 \%)$ : in one case (patient $\mathrm{F}$ ), the retroperitoneal metastasis was classified as 'Hypermethylated', whereas the primary tumor was not; conversely, in the other case (patient $\mathrm{H}$ ), the primary tumor was classified as 'Hypermethylated', whereas the lung metastasis was not (Fig. 3).

\section{Association of intratumor heterogeneity with clinical and genomic features}

The association between intratumor heterogeneity of gene alterations (sequence mutations and copy number alterations in $\mathrm{p} 53 / \mathrm{Rb}$ and $\mathrm{Wnt} / \beta$-catenin pathways) and clinical and genomic features was tested in the 18 patients with at least one gene alteration in one of the two tumor samples.

Intratumor heterogeneity of gene alterations was not significantly associated with the origin of the samples (primary, recurrence or metastasis) used for comparison $(P=0.48)$ or with the delay between surgeries of the matched tumor samples $(P=0.37$, Supplementary Table 3 ).
However, no intratumor heterogeneity was observed in patients for whom the gene alterations profiles were analyzed in two samples from local or metastatic recurrence $(n=4)$.

Intratumor heterogeneity of gene alterations was neither associated with clinical prognostic factors, such as age $(P=0.90)$, ENSAT stage $(P=0.72)$ or Ki67 proliferation index $(P=0.44$, Supplementary Table 3$)$, nor with tumor purity ( 80 vs $88 \%$ in samples without intratumor heterogeneity, $P=0.49)$, nor with other genomic alterations, such as proportion of altered genome (62 vs $38 \%$ in samples without intratumor heterogeneity, $P=0.16$ ), average methylation of four genes (22 vs $11 \%$ in samples without intratumor heterogeneity, $P=0.48)$, chromosome alterations or methylation profiles $(P=1$, data not shown).

\section{Discussion}

Molecular classification has recently emerged as a major prognostic factor in ACC $(7,8,11)$, and targeted molecular markers, based on gene expression, chromosome alterations, methylation or gene alterations, have been proposed to complete the routine prognostic assessment $(9,10,11)$.
This work is licensed under a Creative Commons Attribution-NonCommercial 4.0 International License. ded from Bioscientifica.com at 04/26/2023 11:33:54AM 


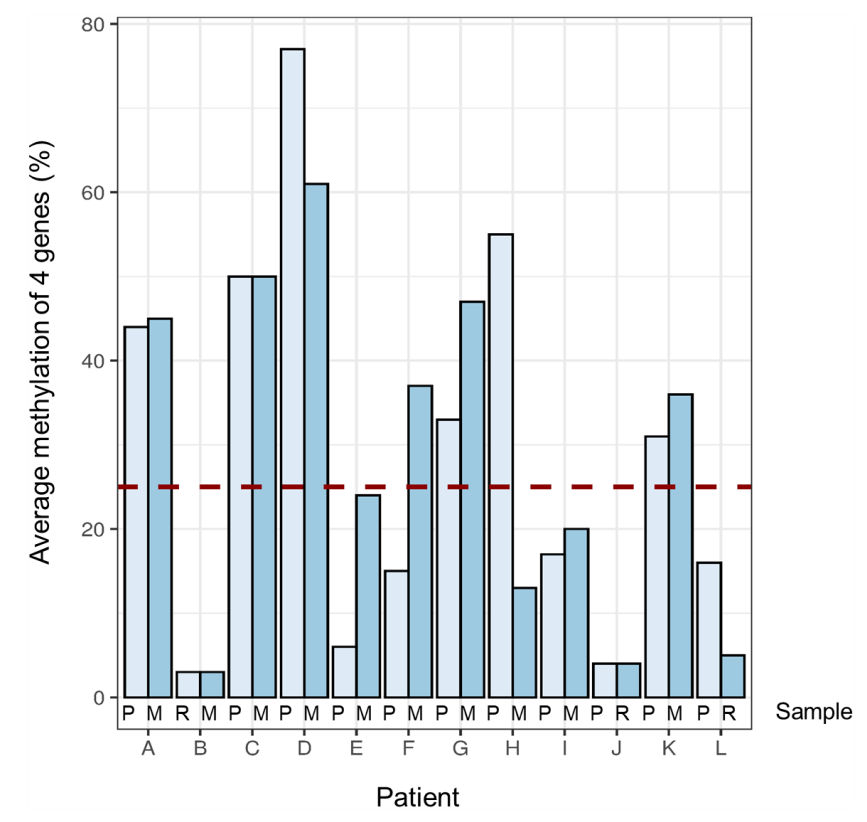

Figure 3

Methylation profiles in two distinct ACC regions (Wuerzburg cohort, 12 patients). The cut-off of $25 \%$ in average methylation of four genes used to discriminate 'Hypermethylated' from 'Non-hypermethylated' $(9,24)$ samples is indicated in dashed red line. $\mathrm{P}$, sample from primary tumor; $\mathrm{R}$, sample from local recurrence; $M$, sample from metastasis.

In the present study, intratumor heterogeneity of targeted chromosome alterations, methylation profiles and gene alterations was evaluated by analyzing two different tumor samples for each patient. Intratumor heterogeneity of gene alterations and methylation profiles was identified in 8/26 (31\%) and 2/12 (17\%) patients, respectively. Intratumor heterogeneity was observed in the comparison of primary/local or metastatic recurrence and primary/primary samples, but not in that of two recurrence sites $(n=4)$. Conversely, the prognostic classification based on chromosome alteration profiles was identical in the two tumor samples for all patients studied.

Strikingly, intratumor heterogeneity of gene alterations was observed in genes usually considered as drivers of adrenocortical tumorigenesis, such as TP53, CTNNB1 and ZNRF3. Indeed, alterations of these genes are almost mutually exclusive, found each in $20 \%$ of ACC $(7,8,26)$. Germline mutations of TP53 are also known to predispose to ACC in part of Li Fraumeni syndrome (27). Moreover, the role of CTNNB1 mutations in adrenocortical tumorigenesis has been validated by functional experiments in cell lines and mouse models $(28,29)$. Therefore, intratumor heterogeneity of such gene alterations appears to go against the hypothesis of early clonal events that drive the tumorigenesis.
Several explanations can be raised to conciliate our findings with this hypothesis. First, p53/Rb or Wnt/ $\beta$-catenin alterations could be drivers of aggressive subclones, associated with metastatic spread and therefore predominant in poor prognosis tumors (30, 31). In this hypothesis, random sampling may have led to underestimating the frequency of such alterations in primary tumor compared to local recurrence and metastasis. In ACC, data regarding intratumor heterogeneity are scarce. Vatrano et al. compared the mutational profile from matched primary and recurrent/ metastatic samples in 10 ACC using targeted NGS and found a high degree of intratumor heterogeneity, extended to prognostic-associated genes, such as TP53, CTNNB1 and ZNRF3 (32). Recently, Gara et al. reported a series of multiregion exome sequencing in 14 ACC patients (15) and observed a 37-57\% overlap in genes that are mutated among different metastatic sites within the same patient. Focusing on genes associated with prognostic value, no intratumor heterogeneity was observed for CTNNB1 and CDKN2A, and no alteration of TP53 and ZNRF3 was found in this study. However, unlike in our study, no comparison with the primary tumor was performed. Of note, gene alterations were explored in two different recurrence sites for four patients in our study. Two of them exhibited gene alterations in $\mathrm{p} 53 / \mathrm{Rb}$ or $\mathrm{Wnt} / \beta$-catenin pathways, with no intratumor heterogeneity (Fig. 1), which is in line with the hypothesis of driver mutations of the aggressive subclone. Our results are also consistent with the literature in other cancer types, in which intratumor heterogeneity has been fully revisited by the advances of genomics in the last decade. Gerlinger et al. have performed exome sequencing in up to 14 different tumor regions of patients with metastatic renal cell carcinoma (12) and showed that only one-third of somatic mutations are shared by all tumor regions. Interestingly, intratumor heterogeneity was also observed for genes recurrently altered and considered as 'drivers' of the disease, such as MTOR and SETD2.

Several pan-cancer studies support a variable chronological timing of CTNNB1 mutations $(33,34)$. In the TCGA ACC study, 5/9 CTNNB1 mutations were late - that is occurring after whole-genome doubling - or subclonal events (8). Conversely, TP53 mutations are usually described as early clonal events $(34,35)$. In a whole-exome analysis of paired primary tumors and metastases in 136 colorectal, lung or breast cancer patients, almost all TP53 mutations (79/85, 93\%) were fully clonal (35). In the TCGA ACC study, all 7 TP53 mutations occurred before whole-genome doubling (8). In the present study, 2/6 TP53 mutations showed

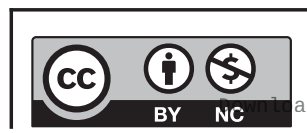

This work is licensed under a Creative Commons Attribution-NonCommercial 4.0 International License. ded from Bioscientifica.com at 04/26/2023 11:33:54AM 
intratumor heterogeneity. A similar result was reported by Vatrano et al. showing 3/7 private TP53 mutations in matched primary and recurrent/metastatic samples (32). These results could be random due to the small number of patients, could suggest a technical artifact - for instance, all heterogeneous TP53 mutations were found in FFPE samples - or unique biology in ACC.

Finally, the hypothesis of revertant alleles may explain some cases of intratumor heterogeneity with alterations in 'driver' genes found in the primary tumor, but not in recurrence. Some deletions of BRCA2 mutation with the restoration of the open reading frame have indeed been described as a mechanism of resistance to therapy in ovarian cancers (36).

Regarding targeted methylation markers, the prognostic classification was unstable for $2 / 12$ patients in our cohort. CpG islands methylator phenotype (CIMP) was described in $50 \%$ of ACC and associated with poor prognosis $(24,37)$. This hypermethylated phenotype was also observed in other tumor types, such as colon cancer, paraganglioma and glioblastoma. In the last two cases, CIMP is related to the accumulation of an oncometabolite due to mutations in genes coding for metabolic enzymes, whereas in ACC the origin of CIMP is still unknown. In a study evaluating the methylation profiles of four different tumor regions in glioblastoma patients (17), the global methylation profile, corresponding to IDH mutational status and its association to CIMP, was stable across the different tumor regions of the same patient. However, the methylation subclass varied from one tumor sample to another in nearly half of patients studied (17). In ACC, different levels of methylation were described among CIMP tumors $(8,37)$. This suggests a progressive rather than binary process and may explain the two cases of intratumor heterogeneity of methylation pattern in our cohort. In the present study, we chose to focus on the fourgene methylation marker that was previously validated in frozen and FFPE samples $(9,24)$. Targeted assessment of GOS2 methylation represents another prognostic marker in ACC (10). Whether this marker would be subject to intratumor heterogeneity remains to be determined.

Limitations of this study include the heterogeneity of the population study, mixing different sample comparisons - mostly primary -recurrence, but also primary-primary, and recurrence-recurrence comparisons - and different assessments of targeted markers in Cochin and Wuerzburg cohorts, resulting in a small number of patients in each analysis. Therefore, the statistical power for the confrontation of mutational status with clinical and genomic features was limited. Moreover, prognostic markers based on gene expression were not evaluated in this study due to the absence or insufficient amount of frozen tissue sample for RNA extraction. DNA-based markers were also assessed with different techniques, on frozen or FFPE samples, based on the local availability of tissue material and techniques. For instance, ZNRF3 mutations were identified by Sanger sequencing in 20/52 samples, whereas NGS was used for all remaining samples and other genes. Since Sanger sequencing has a lower sensitivity than NGS for detecting variants at low allelic ratio, this could have led to overestimating intratumor heterogeneity in patients with ZNRF3 mutations. However, only one patient was classified with intratumor heterogeneity based on the sole results of Sanger sequencing (Fig. 1). Finally, our study could suffer from selection bias, as all patients were treated in referral centers, and most were reoperated for the local or metastatic recurrence. Thus, the study patients may not reflect the general ACC population. For these reasons, our results are not definitive and should be validated in a larger series.

Overall, our study shows that targeted molecular markers based on the pan-genomic profile, that is methylation and chromosome alteration profiles, seem more stable within different ACC regions than the gene alterations profile, and therefore more suitable for prognostic assessment. In a clinical perspective, intratumor heterogeneity of prognostic molecular markers must also be related to that observed for histoprognostic markers, such as Ki67 proliferation index (29). Thus, combining several molecular markers with clinical and pathological prognostic features remains the safest way to produce a robust prognostic classification $(9,11)$. Finally, intratumor heterogeneity of gene alterations should be taken into account for future therapeutics of ACC. With the recent development of cell cycle and Wnt pathway inhibitors $(38,39)$, alterations of $\mathrm{p} 53 / \mathrm{Rb}$ and Wnt/ $\beta$-catenin pathways could be considered as druggable targets in ACC in the near future. The present study suggests that the mutational status should be evaluated in progressive metastatic disease rather than in primary tumor for theranostic purpose.

In conclusion, intratumor heterogeneity was observed in 8/26 and 2/12 of ACC patients for targeted gene alterations profile and targeted methylation profile, respectively, but not for targeted chromosome alteration profile. Combination of multiple targeted molecular markers, along with clinical features, should be preferred to gene alterations profile alone for the prognostic assessment of ACC.

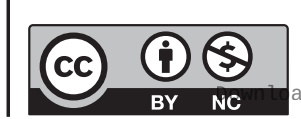

This work is licensed under a Creative Commons Attribution-NonCommercial 4.0 International License. ded from Bioscientifica.com at 04/26/2023 11:33:54AM 


\section{Supplementary materials}

This is linked to the online version of the paper at https://doi.org/10.1530/ EC-20-0228.

\section{Declaration of interest}

The authors declare that there is no conflict of interest that could be perceived as prejudicing the impartiality of the research reported.

\section{Funding}

This work was supported by the Programme de Recherche Translationnelle en Cancérologie to the COMETE network (PRT-K COMETE-TACTIC), the ITMO Cancer AVIESAN (Alliance pour les Sciences de la Vie et de la Santé) as part of the Plan Cancer 2014-2019 (A J received a PhD grant), the Deutsche Krebshilfe (70112969 to C L R and M F) and Deutsche Forschungsgemeinschaft (\#314061271 - CRC/TRR 205) to M F.

\section{Author contribution statement}

$G$ A and J B jointly supervised the work. A J, M F, C L R, J B and G A designed the whole study. S G and B D collected the samples in Cochin cohort. M S carried out the pathology review in Cochin cohort. A J, J L, M F, S A, R L, L G, $C L R, G A$ and J B collected the clinical data. Samples handling was performed by A J, J L and K P. A J, J L, B D, L V, A S, M N, S A, C L R and G A performed the bioinformatic and statistical analyses. Manuscript writing was carried out by A J, J L, M F, S A, C L R, G A and J B. All authors contributed to the manuscript revision.

\section{Acknowledgements}

The authors are grateful to Sonja Steinhauer for the expert technical support and to Michaela Haaf and Martina Zink for coordinating the ENSAT Registry and adrenal biobank in Wuerzburg.

\section{References}

1 Ayala-Ramirez M, Jasim S, Feng L, Ejaz S, Deniz F, Busaidy N, Waguespack SG, Naing A, Sircar K, Wood CG, et al. Adrenocortical carcinoma: clinical outcomes and prognosis of 330 patients at a tertiary care center. European Journal of Endocrinology 2013169 891-899. (https://doi.org/10.1530/EJE-13-0519)

2 Fassnacht M, Johanssen S, Quinkler M, Bucsky P, Willenberg HS, Beuschlein F, Terzolo M, Mueller HH, Hahner S, Allolio B, et al. Limited prognostic value of the 2004 International Union Against Cancer staging classification for adrenocortical carcinoma: proposal for a revised TNM Classification. Cancer 2009115 243-250. (https:// doi.org/10.1002/cncr.24030)

3 Miller BS, Gauger PG, Hammer GD, Giordano TJ \& Doherty GM. Proposal for modification of the ENSAT staging system for adrenocortical carcinoma using tumor grade. Langenbeck's Archives of Surgery 2010395 955-961. (https://doi.org/10.1007/s00423-0100698-y)

4 Beuschlein F, Weigel J, Saeger W, Kroiss M, Wild V, Daffara F, Libé R, Ardito A, Al Ghuzlan A, Quinkler M, et al. Major prognostic role of Ki67 in localized adrenocortical carcinoma after complete resection. Journal of Clinical Endocrinology and Metabolism 2015100 841-849. (https://doi.org/10.1210/jc.2014-3182)

5 Libé R, Borget I, Ronchi CL, Zaggia B, Kroiss M, Kerkhofs T, Bertherat J, Volante M, Quinkler M, Chabre O, et al. Prognostic factors in stage III-IV adrenocortical carcinomas (ACC): an European Network for the Study of Adrenal Tumor (ENSAT) study. Annals of
Oncology 201526 2119-2125. (https://doi.org/10.1093/annonc/ mdv329)

6 Fassnacht M, Dekkers OM, Else T, Baudin E, Berruti A, Krijger R de, Haak HR, Mihai R, Assie G \& Terzolo M. European society of endocrinology clinical practice guidelines on the management of adrenocortical carcinoma in adults, in collaboration with the European Network for the Study of Adrenal Tumors. European Journal of Endocrinology 2018179 G1-G46. (https://doi.org/10.1530/EJE-180608)

7 Assié G, Letouzé E, Fassnacht M, Jouinot A, Luscap W, Barreau O, Omeiri H, Rodriguez S, Perlemoine K, René-Corail F, et al. Integrated genomic characterization of adrenocortical carcinoma. Nature Genetics 201446 607-612. (https://doi.org/10.1038/ng.2953)

8 Zheng S, Cherniack AD, Dewal N, Moffitt RA, Danilova L, Murray BA, Lerario AM, Else T, Knijnenburg TA, Ciriello G, et al. Comprehensive pan-genomic characterization of adrenocortical carcinoma. Cancer Cell 201629 723-736. (https://doi.org/10.1016/j.ccell.2016.04.002)

9 Lippert J, Appenzeller S, Liang R, Sbiera S, Kircher S, Altieri B, Nanda I, Weigand I, Gehrig A, Steinhauer S, et al. Targeted molecular analysis in adrenocortical carcinomas: a strategy toward improved personalized prognostication. Journal of Clinical Endocrinology \& Metabolism 2018103 4511-4523. (https://doi.org/10.1210/jc.201801348)

10 Mohan DR, Lerario AM, Else T, Mukherjee B, Almeida MQ, Vinco M, Rege J, Mariani BMP, Zerbini MCN, Mendonca BB, et al. Targeted assessment of GOS2 methylation identifies a rapidly recurrent, routinely fatal molecular subtype of adrenocortical carcinoma. Clinical Cancer Research 201925 3276-3288. (https://doi. org/10.1158/1078-0432.CCR-18-2693)

11 Assié G, Jouinot A, Fassnacht M, Libé R, Garinet S, Jacob L, Hamzaoui N, Neou M, Sakat J, La Villéon B de, et al. Value of molecular classification for prognostic assessment of adrenocortical carcinoma. JAMA Oncology 2019 [epub]. (https://doi.org/10.1001/ jamaoncol.2019.1558)

12 Gerlinger M, Rowan AJ, Horswell S, Math M, Larkin J, Endesfelder D, Gronroos E, Martinez P, Matthews N, Stewart A, et al. Intratumor heterogeneity and branched evolution revealed by multiregion sequencing. New England Journal of Medicine 2012366 883-892. (https://doi.org/10.1056/NEJMoa1113205)

13 Jamal-Hanjani M, Wilson GA, McGranahan N, Birkbak NJ, Watkins TBK, Veeriah S, Shafi S, Johnson DH, Mitter R, Rosenthal R, et al. Tracking the evolution of non-small-cell lung cancer. New England Journal of Medicine 2017376 2109-2121. (https://doi. org/10.1056/NEJMoa1616288)

14 Li X, Guo X, Li D, Du X, Yin C, Chen C, Fang W, Bian Z, Zhang J, Li B, et al. Multi-regional sequencing reveals intratumor heterogeneity and positive selection of somatic mtDNA mutations in hepatocellular carcinoma and colorectal cancer. International Journal of Cancer 2018143 1143-1152. (https://doi. org/10.1002/ijc.31395)

15 Gara SK, Lack J, Zhang L, Harris E, Cam M \& Kebebew E. Metastatic adrenocortical carcinoma displays higher mutation rate and tumor heterogeneity than primary tumors. Nature Communications 20189 4172. (https://doi.org/10.1038/s41467-018-06366-z)

16 Quek K, Li J, Estecio M, Zhang J, Fujimoto J, Roarty E, Little L, Chow CW, Song X, Behrens C, et al. DNA methylation intratumor heterogeneity in localized lung adenocarcinomas. Oncotarget 20178 21994-22002. (https://doi.org/10.18632/oncotarget.15777)

17 Wenger A, Ferreyra Vega S, Kling T, Bontell TO, Jakola AS \& Carén H. Intratumor DNA methylation heterogeneity in glioblastoma: implications for DNA methylation-based classification. NeuroOncology 201921 616-627. (https://doi.org/10.1093/neuonc/noz011)

18 Weiss LM. Comparative histologic study of 43 metastasizing and nonmetastasizing adrenocortical tumors. American Journal of Surgical Pathology 19848 163-169. (https://doi.org/10.1097/00000478198403000-00001)

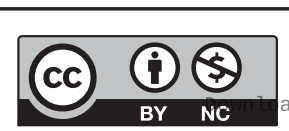

This work is licensed under a Creative Commons Attribution-NonCommercial 4.0 International License. ded from Bioscientifica.com at 04/26/2023 11:33:54AM 
19 Kumar P, Henikoff S \& Ng PC. Predicting the effects of coding nonsynonymous variants on protein function using the SIFT algorithm. Nature Protocols 20094 1073-1081. (https://doi.org/10.1038/ nprot.2009.86)

20 Adzhubei I, Jordan DM \& Sunyaev SR. Predicting functional effect of human missense mutations using PolyPhen-2. Current Protocols in Human Genetics 201376 7.20. (https://doi.org/10.1002/0471142905. hg0720s76)

21 Schwarz JM, Cooper DN, Schuelke M \& Seelow D. MutationTaster2: mutation prediction for the deep-sequencing age. Nature Methods 201411 361-362. (https://doi.org/10.1038/nmeth.2890)

22 Robinson JT, Thorvaldsdóttir H, Winckler W, Guttman M, Lander ES, Getz G \& Mesirov JP. Integrative genomics viewer. Nature Biotechnology 201129 24-26. (https://doi.org/10.1038/nbt.1754)

23 Popova T, Manié E, Stoppa-Lyonnet D, Rigaill G, Barillot E \& Stern MH. Genome Alteration Print (GAP): a tool to visualize and mine complex cancer genomic profiles obtained by SNP arrays. Genome Biology 200910 R128. (https://doi.org/10.1186/gb-2009-10-11-r128)

24 Jouinot A, Assie G, Libe R, Fassnacht M, Papathomas T, Barreau O, Villeon B de la, Faillot S, Hamzaoui N, Neou M, et al. DNA methylation is an independent prognostic marker of survival in adrenocortical cancer. Journal of Clinical Endocrinology and Metabolism 2017102 923-932. (https://doi.org/10.1210/jc.2016-3205)

25 R Core Team. R: A Language and Environment for Statistical Computing. Vienna, Austria: R Foundation for Statistical Computing, 2019. (available at: https://www.R-project.org/)

26 Juhlin CC, Goh G, Healy JM, Fonseca AL, Scholl UI, Stenman A, Kunstman JW, Brown TC, Overton JD, Mane SM, et al. Whole-exome sequencing characterizes the landscape of somatic mutations and copy number alterations in adrenocortical carcinoma. Journal of Clinical Endocrinology and Metabolism 2015100 E493-E502. (https:// doi.org/10.1210/jc.2014-3282)

27 Li FP, Fraumeni JF, Mulvihill JJ, Blattner WA, Dreyfus MG, Tucker MA \& Miller RW. A cancer family syndrome in twenty-four kindreds. Cancer Research 198848 5358-5362.

28 Tissier F, Cavard C, Groussin L, Perlemoine K, Fumey G, Hagneré AM, René-Corail F, Jullian E, Gicquel C, Bertagna X, et al. Mutations of beta-catenin in adrenocortical tumors: activation of the Wnt signaling pathway is a frequent event in both benign and malignant adrenocortical tumors. Cancer Research 200565 7622-7627. (https:// doi.org/10.1158/0008-5472.CAN-05-0593)

29 Berthon A, Sahut-Barnola I, Lambert-Langlais S, Joussineau C de, Damon-Soubeyrand C, Louiset E, Taketo MM, Tissier F, Bertherat J, Lefrançois-Martinez AM, et al. Constitutive beta-catenin activation induces adrenal hyperplasia and promotes adrenal cancer development. Human Molecular Genetics 201019 1561-1576. (https:// doi.org/10.1093/hmg/ddq029)
30 Gaujoux S, Tissier F, Groussin L, Libé R, Ragazzon B, Launay P, Audebourg A, Dousset B, Bertagna X \& Bertherat J. Wnt/beta-catenin and $3^{\prime}, 5^{\prime}$-cyclic adenosine $5^{\prime}$-monophosphate/protein kinase A signaling pathways alterations and somatic beta-catenin gene mutations in the progression of adrenocortical tumors. Journal of Clinical Endocrinology and Metabolism 200893 4135-4140. (https:// doi.org/10.1210/jc.2008-0631)

31 Libè R, Groussin L, Tissier F, Elie C, René-Corail F, Fratticci A, Jullian E, Beck-Peccoz P, Bertagna X, Gicquel C, et al. Somatic TP53 mutations are relatively rare among adrenocortical cancers with the frequent 17p13 loss of heterozygosity. Clinical Cancer Research 200713 844-850. (https://doi.org/10.1158/1078-0432.CCR06-2085)

32 Vatrano S, Volante M, Duregon E, Giorcelli J, Izzo S, Rapa I, Votta A, Germano A, Scagliotti G, Berruti A, et al. Detailed genomic characterization identifies high heterogeneity and histotype-specific genomic profiles in adrenocortical carcinomas. Modern Pathology 201831 1257-1269. (https://doi.org/10.1038/s41379-018-0042-6)

33 McGranahan N, Favero F, Bruin EC de, Birkbak NJ, Szallasi Z \& Swanton C. Clonal status of actionable driver events and the timing of mutational processes in cancer evolution. Science Translational Medicine 20157 283ra54. (https://doi.org/10.1126/scitranslmed. aaa1408)

34 Gerstung M, Jolly C, Leshchiner I, Dentro SC, Gonzalez S, Rosebrock D, Mitchell TJ, Rubanova Y, Anur P, Yu K, et al. The evolutionary history of 2,658 cancers. Nature $2020 \mathbf{5 7 8} 122-128$. (https://doi.org/10.1038/s41586-019-1907-7)

$35 \mathrm{Hu}$ Z, Li Z, Ma Z \& Curtis C. Multi-cancer analysis of clonality and the timing of systemic spread in paired primary tumors and metastases. Nature Genetics 202052 701-708. (https://doi. org/10.1038/s41588-020-0628-z)

36 Edwards SL, Brough R, Lord CJ, Natrajan R, Vatcheva R, Levine DA, Boyd J, Reis-Filho JS \& Ashworth A. Resistance to therapy caused by intragenic deletion in BRCA2. Nature 2008451 1111-1115. (https:// doi.org/10.1038/nature06548)

37 Barreau O, Assié G, Wilmot-Roussel H, Ragazzon B, Baudry C, Perlemoine K, René-Corail F, Bertagna X, Dousset B, Hamzaoui N, et al. Identification of a CpG island methylator phenotype in adrenocortical carcinomas. Journal of Clinical Endocrinology and Metabolism 201398 E174-E184. (https://doi.org/10.1210/jc.20122993)

38 Ingham M \& Schwartz GK. Cell-cycle therapeutics come of age. Journal of Clinical Oncology 201735 2949-2959. (https://doi. org/10.1200/JCO.2016.69.0032)

39 Haseeb M, Pirzada RH, Ain QU \& Choi S. Wnt signaling in the regulation of immune cell and cancer therapeutics. Cells 20198 1380. (https://doi.org/10.3390/cells8111380)

Received in final form 9 June 2020

Accepted 1 July 2020

Accepted Manuscript published online 2 July 2020 https://ec.bioscientifica.com https://doi.org/10.1530/EC-20-0228 (c) 2020 The authors Published by Bioscientifica Ltd

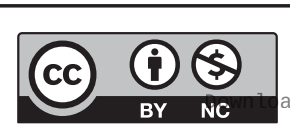

This work is licensed under a Creative Commons Attribution-NonCommercial 4.0 International License. ded from Bioscientifica.com at 04/26/2023 11:33:54AM 